\title{
Longitudinally extensive transverse myelitis: a rare association with common variable immunodeficiency
}

\author{
Edwin Jabbari, ${ }^{1}$ Charles Robert Marshall, ${ }^{2}$ Hilary Longhurst, ${ }^{3}$ \\ Richard Sylvester ${ }^{4}$
}

${ }^{1}$ Department of Neurology, Homerton University Hospital NHS Trust, London, UK ${ }^{2}$ Department of Neurology, Barts and The London Hospital NHS Trust, London, UK

${ }^{3}$ Department of Immunology, Barts and The London NHS

Trust, London, UK

${ }^{4}$ Homerton University Hospital NHS Trust, National Hospital of Neurology and Neurosurgery, $\mathrm{UCH}$, London, UK

\section{Correspondence to}

Dr Richard Sylvester, Homerton University Hospital NHS Trust,

National Hospital of Neurology and Neurosurgery, UCH, London, UK;

richard.sylvester@nhs.net

Accepted 29 July 2014 Published Online First

21 October 2014

CrossMark

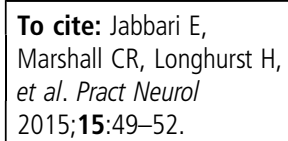

\section{INTRODUCTION}

Transverse myelitis requires careful investigation, as although many causes respond acutely to immunomodulation, the longer term management depends upon its precise cause. We describe a patient presenting acutely with a corticosteroidresponsive longitudinally extensive transverse myelitis and granulomatous lung lesions with a previous history of recurrent generalised lymphadenopathy, pyogenic infections and idiopathic thrombocytopenic purpura. We initially suspected an underlying primary autoimmune disorder. However, investigations showed granulomatous common variable immunodeficiency (gCVID) and following regular intravenous immunoglobulin, her symptoms did not recur. We discuss this relatively common immunodeficiency disease, frequently misdiagnosed as a systemic autoimmune disease (often sarcoidosis). We also discuss the range of neurological syndromes, including transverse myelitis, that may accompany common variable immunodeficiency (CVID).

\section{CASE REPORT}

A 41-year-old Caucasian woman presented with a 1-week history of progressive bilateral leg weakness, with urinary hesitancy and frequency. She had an intriguing past medical history of multiple episodes of corticosteroid-responsive widespread tender lymphadenopathy over 10 years, splenectomy for refractory idiopathic thrombocytopenic purpura in 2005 , frequent respiratory tract infections and multiple buttock abscesses.

On examination at presentation, there was increased tone in both lower limbs with mild bilateral proximal weakness. Her reflexes were pathologically brisk in both legs and plantars were extensor. There were no sensory abnormalities.
Upper limb and cranial nerve examinations were normal.

Routine blood tests were normal, including full blood count, renal function, liver function, clotting function and inflammatory markers. Her MR scan of brain was normal, but an MR scan of spine showed longitudinally extensive cord signal change between $\mathrm{T} 2$ and the conus medullaris (figure 1) with focal contrast enhancement at T6/7 (figure 2), consistent with transverse myelitis. Further investigation was aimed at finding the cause from a wide differential (table 1).

Her cerebrospinal fluid (CSF) was acellular with a mildly elevated protein of $0.56 \mathrm{~g} / \mathrm{L}(0.15-0.45)$, normal glucose and negative oligoclonal bands. Contrast-enhanced CT imaging showed extensive mediastinal and abdominal lymphadenopathy with bronchiectactic and nodular changes throughout both lung fields (figure 3), raising the possibility of sarcoidosis, although her serum ACE was normal at $33 \mathrm{U} / \mathrm{L}$ (25-82). Subsequent lymph node biopsy showed no evidence of lymphoma or granulomatous disease but did find necrotising lymphadenitis with CD68 histiocytes. Biopsy immunostaining was negative for Brucella, Trichinella, hydatid and toxoplasma. A transbronchial biopsy with bronchial washings showed only non-specific chronic inflammation. Other negative investigations included: HIV serology and p24 antigen; Mantoux and QuantiFERON-TB test; autoimmune profile, including antinuclear antibodies, dsDNA, extractable nuclear antigen and anti-aquaporin-4 antibodies and blood/ CSF testing for bacterial and viral organisms. including varicella zoster, Lyme and Brucella by PCR. Immunoglobulin testing showed a low level of IgG at $1.8 \mathrm{~g} / \mathrm{L}$ (6.0-13.0), IgA at $0.34 \mathrm{~g} / \mathrm{L}(0.8-3.0)$ and normal of IgM at $0.44 \mathrm{~g} / \mathrm{L}(0.4-2.5)$. 
She responded well to a 3-day course of high-dose intravenous methylprednisolone and fully recovered from her neurological symptoms after rehabilitation. Repeat MR scan of cord some months later showed less intrinsic cord signal change and no focal enhancement.

Further investigation of her hypogammaglobulinaemia, including lymphocyte subsets (normal CD4 and CD8 T cells, minimally elevated CD19 B cell and CD56 NK cell numbers), B lymphocyte subpopulations and functional antibody responses to immunisations, suggested a diagnosis of CVID (granulomatous form). Peripheral blood B cells showed a phenotype typical of gCVID with low $(0.2 \%$ of lymphocytes) switched memory B cells (CD19, CD27, IgM-), normal range $>2 \%$, with a high proportion $(52.5 \%)$ of $\mathrm{B}$ cells expressing CD21 (normal range <10\%), a marker of

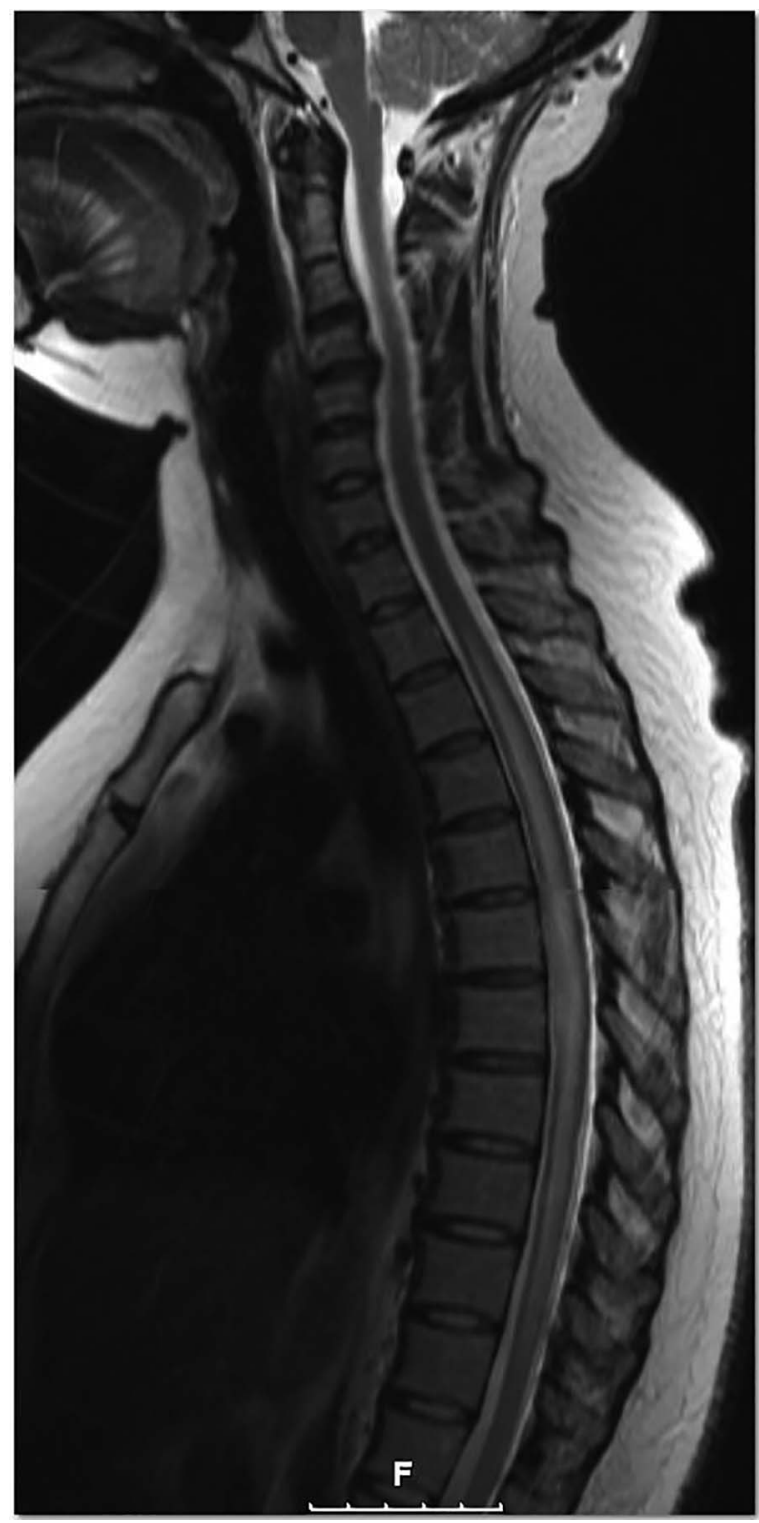

Figure 1 T2-weighted sagittal MR scan of spine showing longitudinally extensive cord signal change between $\mathrm{T} 2$ and the conus medullaris.

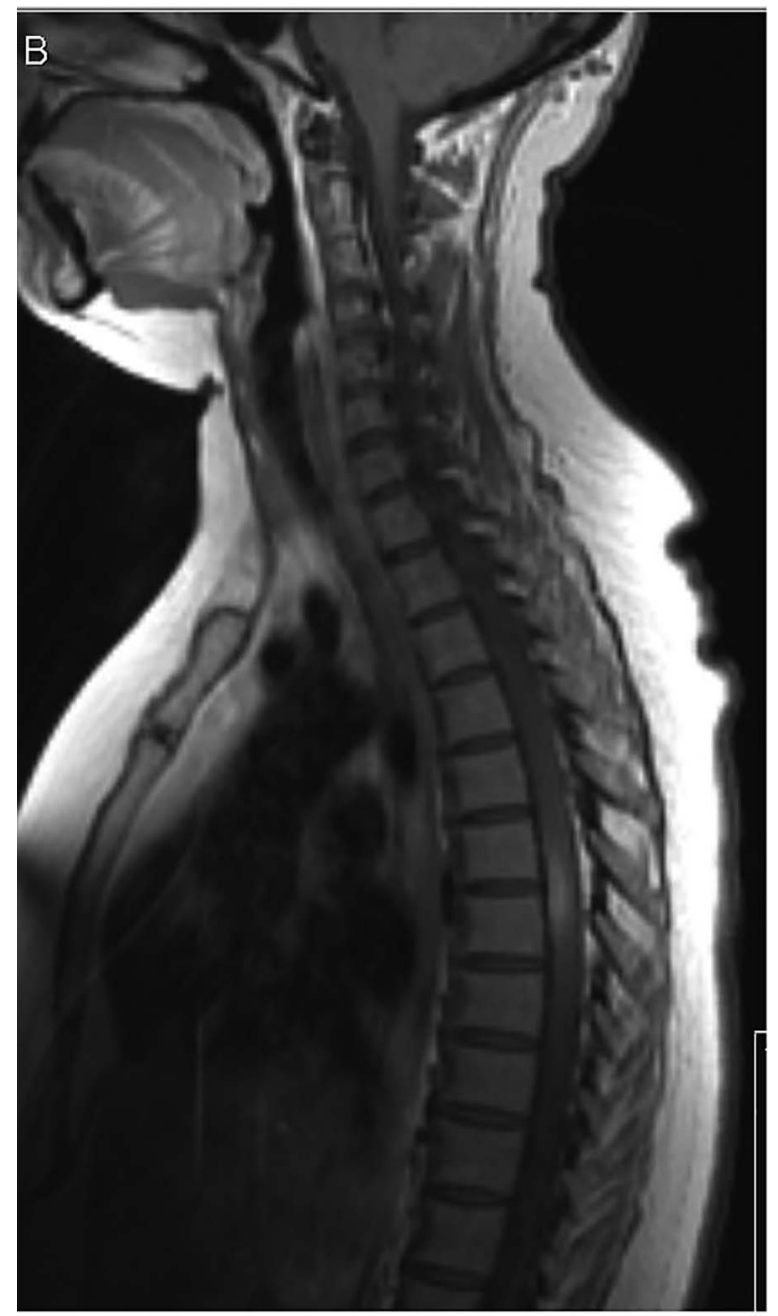

Figure 2 Post-contrast T1-weighted sagittal MR scan of spine showing focal enhancement at T6/7.

autoimmunity in CVID. There were inadequate antibody responses to test vaccinations with Prevenar 13 (Pneumococcus) and tetanus toxoid. Haemophilus antibodies were protective but did not increase postvaccination. The CD68 histiocytes seen previously on lymph node biopsy were a marker of activated polyclonal macrophages, an expected finding in gCVID.

She subsequently responded successfully to replacement dose $(0.5 \mathrm{~g} / \mathrm{kg} / 3$ weeks $)$ of intravenous immunoglobulin and currently has had no recurrence of her neurological or systemic symptoms.

\section{DISCUSSION}

CVID is the most common symptomatic primary immunodeficiency (table 2), with a prevalence between 1:2500 and 1:50 000 in the general population. ${ }^{1}$

The cause is unknown, but hypotheses include the role of disease susceptibility genes, such as transmembrane activator and calcium-modulator and cyclophilinligand interactor ${ }^{2}$ and subsequent environmental stimuli, such as cytomegalovirus infection. ${ }^{3}$ Its common clinical features (table 3) include autoimmunity and 
Table 1 Causes of longtitudinally extensive transverse myelitis

\begin{tabular}{lll}
\hline Aetiology & Diagnosis & Diagnostic markers \\
\hline Infective & Bacterial, eg, Lyme disease, tuberculosis and syphilis & Serology, culture and PCR on blood and CSF \\
& Viral, eg, herpes virus, HIV & Serology and PCR on blood and CSF \\
Inflammatory & Multiple sclerosis & MRI brain; positive CSF oligoclonal bands \\
& Neuromyelitis optica (NMO) & Positive serum NMO IgG \\
& Sarcoidosis & Raised serum ACE/urinary calcium; granulomas on lymph node biopsy \\
& Systemic lupus erythematosus & Positive anti-nuclear antibody, anti-dsDNA and anti-phospholipid antibodies \\
& Sjögren's syndrome & Positive anti-nuclear antibody, anti-Ro and anti-La antibodies; lip biopsy \\
Neoplastic & CNS tumours, eg, astrocytoma, ependymoma & MRI, biopsy \\
& Paraneoplastic & Positron emission tomography scan, onconeuronal antibodies \\
Metabolic & Vitamin B Bi2 deficiency & Serum B 12 $_{12} /$ methylmalonic acid levels \\
& Copper deficiency & Serum copper levels \\
Vascular & Spinal cord infarction & MRI, history \\
& Spinal cord arteriovenous malformation & MRI/angiogram \\
Trauma & Spinal cord contusion & MRI, history \\
\hline
\end{tabular}

CNS, central nervous system.

granulomatous inflammation, which may be misdiagnosed as sarcoidosis, especially when lymphadenopathy is present. ${ }^{4}$

Recurrent lymphadenopathy and bacterial infections coupled with hypogammaglobulinaemia and a coexisting autoimmune history (idiopathic thrombocytopenic purpura) raises the suspicion of an underlying diagnosis of CVID. The diagnosis is criteria based, involving low immunoglobulins and impaired functional antibody responses. ${ }^{5}$ Therefore, a diagnosis of granulomatous form of CVID is an ill-defined subset of this condition. This is further supported by the fact that all CVID patients can have granulomatous, autoimmune and inflammatory phenomena, which are all probably inter-related and the consequence of a dysfunctional immune response to persistent infection. Immunoglobulin replacement prevents infections and currently is the mainstay of therapy for CVID. ${ }^{5}$ Immunoglobulin replacement does not reliably reverse autoimmune or inflammatory complications and so corticosteroids or other immunosuppressive drugs are required, although evidence of benefit is lacking. ${ }^{5}$

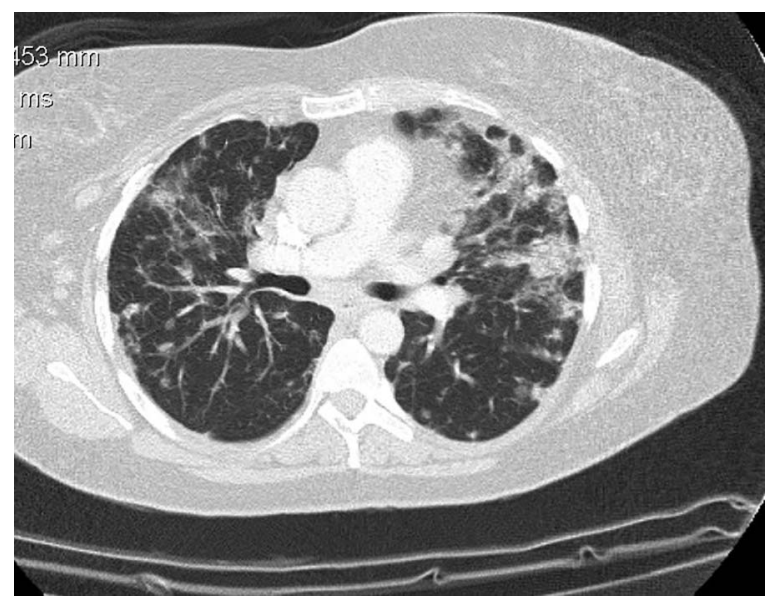

Figure 3 Post-contrast axial CT scan of chest showing bilateral lung nodules and traction bronchiectasis.
Neurological features in CVID are rare. However, several reported cases, taken together, suggest that this is a true association. Initial cases of transverse myelitis associated with CVID occurred among a case series of encephalomyelitis in primary hypogammaglobulinaemia. ${ }^{6}$ Subsequent cases of transverse myelitis have also shown this association, as well as other neurological manifestations such as cognitive impairment, ataxia, spasticity, weakness ${ }^{7}$ and bilateral optic neuritis. ${ }^{8}$ As in this case, almost all of the patients reported previously responded well clinically to a combination of corticosteroids and intravenous immunoglobulin with no recurrences. Hypotheses for neurological involvement in CVID include a possible autoimmune reaction against neuronal tissue, ${ }^{7}$ a yet undefined infectious agent $^{7}$ and Vitamin E deficiency due to an associated enteropathy, ${ }^{9}$ although none have yet been proven. This is evidently an area of ongoing research with further surveillance of CVID patients recommended. Furthermore, myelitis is a recognised feature of secondary immunodeficiency states such as HIV. However, its association with other primary immunodeficiency syndromes has been limited to infective causes, such as coxsackie virus, ${ }^{10}$ secondary to the

Table 2 Causes of immunodeficiency

\begin{tabular}{|c|c|c|}
\hline \multirow[t]{3}{*}{ Primary } & $\begin{array}{l}\text { Antibody deficiency } \\
\text { syndromes }\end{array}$ & $\begin{array}{l}\text { Common variable } \\
\text { immunodeficiency } \\
\text { X linked agammaglobulinaemia } \\
\text { Selective IgA deficiency }\end{array}$ \\
\hline & Cell-mediated deficiency & DiGeorge syndrome \\
\hline & & $\begin{array}{l}\text { Severe combined } \\
\text { immunodeficiency }\end{array}$ \\
\hline \multirow[t]{5}{*}{ Secondary } & $\begin{array}{l}\text { Malnutrition } \\
\text { Major trauma/surgery }\end{array}$ & \\
\hline & Lymphoreticular & Lymphoma \\
\hline & malignancy & $\begin{array}{l}\text { Leukaemia } \\
\text { Myeloma }\end{array}$ \\
\hline & Drugs & $\begin{array}{l}\text { Cytotoxic treatment } \\
\text { Immunosuppressant treatment }\end{array}$ \\
\hline & Viral & HIV \\
\hline
\end{tabular}


Table 3 Common features of common variable immunodeficiency

\begin{tabular}{ll}
\hline Infections & Sinopulmonary infections \\
& Bacterial gastroenteritis \\
& Cellulitis/skin abscesses \\
& Bacterial meningitis \\
& Asthma \\
& Bronchiectasis \\
Pulmonary disease & Interstitial lung disease \\
& Inflammatory bowel disease \\
& Gastrointestinal lymphoma \\
Gastrointestinal disease & Bacterial overgrowth \\
& Lungs \\
& Lymph nodes \\
Granulomatous disease & Intestine \\
& Central nervous system \\
& Haemolytic anaemia and idiopathic \\
& thrombocytopenic purpura \\
Autoimmunity & Rheumatoid arthritis \\
& Pernicious anaemia \\
& Non-Hodgkin's lymphoma \\
& Gastrointestinal cancers \\
Malignancy & Lymphadenopathy \\
Others & Splenomegaly \\
& Fever/night sweats
\end{tabular}

immunodeficient state rather than a direct consequence of the condition itself.

With regards to treatment, intravenous immunoglobulin and corticosteroid-related failures are rare in CVID. This has led to increasing interest in the role of the pro-inflammatory cytokine, tumour necrosis factor (TNF)- $\alpha$ and the use of TNF- $\alpha$ inhibitors such as infliximab. In relation to neurological involvement in CVID, infliximab has had mixed results. Reports suggest that a synergistic effect of intravenous immunoglobulin and infliximab improves neuroimaging appearances as well as systemic features such as fever, night sweats and lymphadenopathy whilst having no effect clinically on neurological deficits. ${ }^{11}$ Furthermore, a recent case of recurrent myelitis in CVID showed a good response to once weekly subcutaneous immunoglobulin with remission at 3-year follow up. ${ }^{12}$

Contributors EJ was the lead writer of the case report. RS reviewed and revised each draft. He also follows the patient up in the outpatient Neurology clinic. CRM was the Neurology registrar who initially reviewed and regularly provided Neurology input, while the patient was an inpatient. He also reviewed and revised the final draft. HL investigated and diagnosed the patient with CVID as an outpatient. She also contributed to the writing of the CVID section of the discussion.

Competing interests None.

Patient consent Obtained.

Provenance and peer review Not commissioned. Externally peer reviewed. This paper was reviewed by Neil Scolding, Bristol, UK.

\section{REFERENCES}

1 Resnick ES, Moshier EL, Godbold JH, et al. Morbidity and mortality in common variable immune deficiency over 4 decades. Blood 2012;119:1650-7.

2 Rachid R, Castiqli E, Geha RS, et al. TACI mutation in common variable immunodeficiency and IgA deficiency. Curr Allergy Asthma Rep 2006;6:357-62.

3 Marashi SM, Raeiszadeh M, Enright V, et al. Influence of cytomegalovirus infection on immune cell phenotypes in patients with common variable immunodeficiency. J Allergy Clin Immunol 2012;129:1349-56.

4 Arnold DF, Wiggins J, Cunningham-Rundles C, et al. Granulomatous disease: distinguishing primary antibody disease from sarcoidosis. Clin Immunol 2008;128:18-22.

5 Bonilla FA, Bernstein L, Khan DA, et al. Practice parameter for the diagnosis and management of primary immunodeficiency. Ann Allergy Asthma Immunol 2005;94:1-63.

6 Rudge P, Webster AD, Revesz T, et al. Encephalomyelitis in primary hypogammaglobulinaemia. Brain 1996;119:1-15.

7 Ziegner UH, Kobayashi RH, Cunningham-Rundles C, et al. Progressive neurodegeneration in patients with primary immunodeficiency disease on IVIG treatment. Clin Immunol 2002;102:19-24.

8 Sempere AP, Tahoces ML, Palao-Duarte S, et al. Bilateral optic neuritis in a 26 year old man with common variable immunodeficiency: a case report. J Med Case Rep 2011;5:319-20.

9 Aslam A, Misbah SA, Talbot K, et al. Vitamin E deficiency induced neurological disease in common variable immunodeficiency: two cases and a review of the literature of vitamin E deficiency. Clin Immunol 2004;112:24-9.

10 Katamura K, Hattori H, Kunishima T, et al. Non-progressive viral myelitis in X-linked agammaglobulinaemia. Brain Dev 2002;24:109-11.

11 Kumar N, Hagan JB, Abraham RS, et al. Common variable immunodeficiency-associated myelitis. Report of treatment with infliximab. J Neurol 2008;255:1821-4.

12 Danieli MG, Pettinari L, Marinangeli L, et al. Recurrent myelitis in common variable immunodeficiency successfully managed with high dose subcutaneous immunoglobulin. BMJ Case Rep 2012;2012:pii:bcr0120125637. 